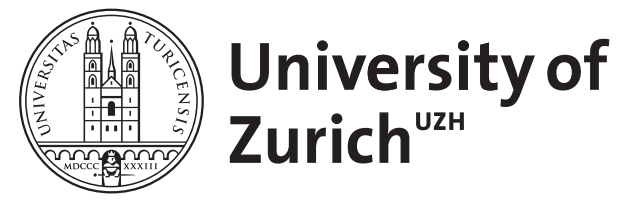

\title{
Zwei Studien zur Intersexualität
}

Streuli, J C

DOI: https://doi.org/10.1007/s00481-011-0169-1

Posted at the Zurich Open Repository and Archive, University of Zurich ZORA URL: https://doi.org/10.5167/uzh-60860

Journal Article

Published Version

Originally published at:

Streuli, J C (2012). Zwei Studien zur Intersexualität. Ethik in der Medizin, 24(2):167-169.

DOI: https://doi.org/10.1007/s00481-011-0169-1 


\title{
Zwei Studien zur Intersexualität
}

\author{
Jürg C. Streuli
}

Online publiziert: 6. Dezember 2011

(C) Springer-Verlag 2011

\begin{abstract}
Allein in Deutschland kommen je nach Definition und Quelle jährlich 100 bis 500 Kinder mit mehrdeutigen biologischen Geschlechtsmerkmalen, auch Intersexualität oder „DSD“ für disorders of sex development genannt, zur Welt. Ein Teil dieser Kinder bedarf einer umgehenden medizinischen Betreuung, um nicht an einem Salzverlust bei hormoneller Entgleisung oder einer Blut- oder Urinvergiftung bei unzureichender Funktion der harn- und stuhlableitenden Wege zu sterben. Weiter besteht bei gewissen Formen ein erhöhtes Risiko, an bösartigen Keimzelltumoren zu erkranken. Für die Betreuung dieser Probleme erlangte die Medizin in den letzten Jahren mit mehreren zentrumsübergreifenden Studien eine zunehmende diagnostische und therapeutische Expertise. Aber allein schon die überwältigende Anzahl von kontroversen Publikationen, Empfehlungen, Selbsthilfe- und Arbeitsgruppen sowie die jüngsten Anstrengungen im deutschen Ethikrat, der schweizerischen Nationalen Ethikkommission oder auch des internationalen Leichtathletikverbands deuten darauf hin, dass damit nur die Spitze des Eisberges umrissen ist. Denn obwohl der „DSD“-Begriff eine möglichst exakte medizinisch-biologische Definition anstrebt, sind neben einer psychologischen und biologischen Ebene immer auch soziale und historische Bedingtheiten miteingeschlossen.

Die hier vorgestellten Bücher von Ulrike Klöppel und Kathrin Zehnder beleuchten diesen Eisberg auch unterhalb der medizinisch-biologischen Oberfläche und tauchen dazu in die dunklen Gewässer der aktuellen und vergangenen medizinischen, gesellschaftlichen und persönlichen Umgangsformen mit Intersexualität.
\end{abstract}

\section{Ulrike Klöppel (2010) XXOXY ungelöst. Hermaphroditismus, Sex und Gender in der deutschen Medizin. Eine historische Studie zur Intersexualität Transcript Verlag, Bielefeld, 698 Seiten, 39,80 €, ISBN 978-3-8376-1343-8}

Ulrike Klöppels Buch lüftet dazu in einem ersten Schritt die Archive der ärztlichen Kunst mit Blick auf den deutschsprachigen Hermaphroditismus-Diskurs. In einem zweiten Schritt

Dr. med. J. C. Streuli $(\triangle)$

Zürich, Schweiz

E-Mail: streuli@ethik.uzh.ch 
stellt sie die folgenschwere Verschmelzung von „Gender“-Konzepten und medizinisch-chirurgischen Behandlungsansätzen ab den 1950er Jahren in einen umfassenden historischen Kontext. Das Resultat beeindruckt sowohl quantitativ als auch inhaltlich. Klöppel gelingt es, langfristige Veränderungen der Kategorie des Geschlechts und ihre Auswirkungen bis in die jüngsten Behandlungsempfehlungen darzulegen. Demzufolge wich die in der hippokratisch-galenischen Tradition vertretene Auffassung von Geschlecht als Kontinuum zwischen männlich und weiblich in der zweiten Hälfte des 18. Jahrhunderts dem Konzept eines strikten Geschlechtsdimorphismus, um im 19. Jahrhundert in Form eines graduellen Stufenmodells erneut die Perspektive auf Menschen mit Intersexualität zu prägen. Neu war jedoch der Einfluss von biologischen Entwicklungsmodellen, welche das im „DSD“-Begriff steckende störungsorientierte Verständnis erst ermöglichten und das ,uneindeutige“ Genitale zu einem ,unvollkommenen“ Genitale werden ließen - ein Verständnis, das gepaart mit einer zunehmend psychosozial-orientierten Perspektive wiederum für die Entwicklung des „Gender“-Konzeptes von großer Bedeutung war. Als Folge entstand ein Behandlungskonzept, das eine frühestmögliche operative, hormonelle und psychosoziale Zuweisung zu einem Geschlecht bei lebenslanger Geheimhaltung der vorab bestehenden Uneindeutigkeit verlangte (optimal gender policy). Wie man heute weiß, kann dies für die Betroffenen auch oder gerade nach Aufdecken ihrer Geschichte eine massive Verunsicherung und Verletzung bedeuten. Im Unterschied zu Stimmen aus den Gender- und Queer-Studies sieht Klöppel die Medizin jedoch weniger als Initiantin der Abwehr von geschlechtlicher Ambivalenz, sondern relativiert vielmehr die medizinische Geltungsmacht gegenüber der Gesellschaft. Hingegen unterstreicht sie das Versäumnis, Patienten und Eltern über Unsicherheiten und Nebenwirkungen von Behandlungsansätzen aufzuklären. Gleichzeitig wird in Klöppels Untersuchungen auch die bis an Unbedarftheit grenzende Flexibilität der Medizin deutlich, wenn es um die Suche nach Lösungen und Konzepten für komplexe Phänomene geht. Der „Gender"-Begriff spielt dabei als historisch konstituiertes Werkzeug zur Begründung von Diagnose und Behandlung direkt oder indirekt eine zentrale Rolle.

Klöppels Verständnis für die vielfältigen Einflussfaktoren, die das individuelle Handeln bestimmen, führt sie schließlich zum Fazit, dass mit Blick auf die Geschlechterdifferenz und Intersexualität für Politik, Recht und Medizin sehr wohl Handlungsspielräume vorhanden sind. Diese, so Klöppel weiter, wurden jedoch mit Blick auf Rahmenbedingungen und Behandlungskonzepte kaum genutzt. Mit diesem Fazit ist eine Einsicht verbunden, welche bisher von Forschungsprojekten, Therapeuten, Arbeitsgruppen und Gesetzgeber nur bedingt beachtet wird: Intersexualität ist nicht nur eine Störung, sondern immer auch ein Rätsel, das letztendlich dem Kind gehört und das weder mit Gewalt aufgedeckt noch verdeckt werden kann.

\section{Kathrin Zehnder (2010) Zwitter beim Namen nennen. Intersexualität zwischen Pathologie, Selbstbestimmung und leiblicher Erfahrung Transcript Verlag, Bielefeld, 448 Seiten, 33,80 €, ISBN 978-3-8376-1398-8}

Genau das wird im zweiten hier zu besprechenden Buch eindrücklich dargestellt. Während Klöppels Arbeit sich primär auf wissenschaftliche Quellen stützt und dementsprechend die Schwierigkeit und Problematik der Medizin im Umgang mit den betroffenen Menschen darstellt, bietet Kathrin Zehnder einen Einblick in die Schwierigkeit und Problematik der betroffenen Menschen im Umgang mit der Medizin. Neben einer umfassenden Übersicht der unterschiedlichen Diskurse analysiert sie den Inhalt des „Hermaphroditen-Forums“, einer Austauschplattform im Internet für Betroffene mit über 20.000 Einträgen und mehr als 1.000 Themen. Ergänzt wurden die Resultate mit den Einträgen zweier Weblogs (Blogs). 
Diese virtuellen Räume, so die Soziologin, erlauben Betroffenen, Distanz und Hemmnisse zu überwinden und sich einen identitätsstiftenden Raum für „Anderes“ zu schaffen. Vor den Augen des Lesers entsteht so ein facettenreicher Gegendiskurs zur therapeutischintervenierenden Perspektive der Fachpersonen. Für Zehnder sind Geschlechter dabei nicht einfach konstruiert, sondern stellen in Anlehnung an Maihofer „Existenzweisen“ dar, welche die Gesellschaft maßgeblich strukturieren. Die Möglichkeit, Intersexualität als Geschlecht bzw. Existenzweise in der Gesellschaft zu leben, wird von Zehnder jedoch kritisch betrachtet und Vorstöße, Intersexualität durch ein striktes Operationsverbot kosmetisch-orientierter Eingriffe zu einer gesellschaftlich sicht- und lebbaren Tatsache werden zu lassen, ergänzt sie mit der Frage, ob es sich beim Leben in einem intersexuellen Körper um ein Dürfen oder ein Müssen handeln soll. Der medizinische Diskurs weiche dieser Frage oftmals mit dem Argument aus, dass es Recht und Pflicht der Eltern sei, für ihr (Klein-)Kind eine Entscheidung zu fällen. Queer- und Gender Studies wiederum tendierten zu einem Rundumschlag gegen die dichotome Geschlechtertrennung und betrachteten die Frage als überflüssig, sobald der gesellschaftliche Druck der Geschlechter wegfällt. Zehnders Einblick in die Welt der Betroffenen relativiert sowohl die elterliche Entscheidungshoheit als auch die Queerorientierten Ansprüche. Problematisch erscheint aus Zehnders Sicht primär die Selbstverständlichkeit, mit der die Medizin eine Problemlösung für das Leben der Betroffenen anbietet, und die mangelhafte Professionalität, mit der die körperlichen Probleme - oftmals als Nebenwirkung der Behandlung - angegangen werden. Aber auch die Verunsicherung und Stigmatisierung als Folge einer medizinischen Behandlung im Kindesalter erscheint in Zehnders Buch durchgehend als existentielles Thema, wie stellvertretend dieser Forenbeitrag zeigt: ,[...] ich zweifle nicht an mir, aber ich bin mit den Zweifeln der Anderen, der Eltern und anderer Menschen, die mit mir umgingen, Groß geworden und ich konnte mich nicht immer gegen die Angriffe auf meine Realität schützen“" (S. 273). In den von Zehnder sichtbar gemachten Forenbeiträgen wird deutlich, wie wichtig es ist, nicht nur die eigene Besonderheit, sondern auch die juristischen, medizinischen und gesellschaftlichen Eingriffe für oder gegen diese Besonderheit positiv in einen persönlichen Lebensentwurf einbauen zu können. Therapeutisch-intervenierende Eingriffe, welche über die eingangs dargestellten vital indizierten Maßnahmen hinausgehen, erscheinen dadurch, unabhängig von den Entscheidungskompetenzen der Eltern, sehr fragwürdig.

Sowohl Klöppel als auch Zehnder verzichten weitgehend auf normative Schlussfolgerungen aus ihren Resultaten und bieten vielmehr wichtige und sich ergänzende Grundlagen für eine weiterführende, dringend notwendige Diskussion über Ethik, Recht und Professionalität.

Durch die Lektüre der beiden Bücher wird deutlich, dass Phänomene wie „Intersexualität“" weit mehr sind als medizin-biologisch und psychologisch definierte „Störungen“. Wer und was den Fachpersonen zu einem bestimmten Zeitpunkt die Rechtfertigung ermöglicht, ihre einschneidenden Maßnahmen anzuwenden, ist vermutlich nur im Zusammenspiel von individuellen und übergeordneten Ebenen zu klären. Als Bindeglied dienen historische und gesellschaftliche Zusammenhänge sowie Wortmeldungen, deren Horizont über die (interdisziplinäre) Sprechstunde in der Spezialklinik hinausreichen. Entsprechend wertvoll sind sozialwissenschaftliche Arbeiten, wie sie hier von Ulrike Klöppel und Kathrin Zehnder vorgelegt wurden. Beide Bücher verlangen von ihren Lesern zwar eine gewisse Offenheit für sozialwissenschaftliche und historische Zugänge sowie etwas Geduld mit der umfangreichen Datenlage. Trotzdem sind die Bücher gerade auch für Fachpersonen aus Medizin, Pflege, Psychologie, Recht und Ethik, die in ihrer Tätigkeit mit Fragestellungen zu Intersexualität und den damit verbundenen Menschen in Kontakt kommen, sehr wertvoll. Sie erhalten durch Klöppels Werk eindrückliche Einblicke in die Quellen ihrer eigenen Handlungen und mit Zehnders Arbeit eine zusätzliche, wichtige Perspektive auf deren mögliche Folgen. 